\title{
The effect of home visits on healthcare resource utilisation
}

Tai-Yin Wu, Sheng-Huang Hsiao, Sheng-Jean Huang, Kuan-Liang Kuo, Lin-Chung Woung, Fang-Chun Chen

\section{Background and objective}

Home visits may change patients' healthcare resource utilisation, including hospital admission, medications, outpatient and emergency room visits. The aim of the present study was to report changes in healthcare resource utilisation after the provision of home healthcare services.

\section{Methods \\ This was a multicentre follow-up study. Data on health and functional statuses were collected during home visits. Electronic medical records of 12 medical institutions were retrieved (outpatient visits, emergency care use, hospital admissions and prescription medications). The researchers analysed healthcare utilisation and medications before and after enrolment.}

\section{Results}

There were 246 participants. The mean age was 85.5 years ( $52 \%$ men). There was an increase in annual outpatient visits and a decrease in hospital admission days after enrolment (13.7-15.3 visits/year and 17.5-15 days/year, respectively). The number of medical institutions visited increased, but specialties and doctors visited decreased. Oral medications also increased (3.3-4.3 types).

\section{Discussion}

Home visits help decrease days of hospital admission, but not medications or outpatient or emergency room visits.
AGEING IN PLACE is one of the ultimate goals in gerontology. Most older people in Taiwan wish to live with their children (66.2\%) or with their spouse (18.6\%). ${ }^{1}$ However, access to necessary healthcare services prevents older people from ageing in place. Homebound individuals are usually poorly served by office-based primary care delivery models, owing to substantial accessibility issues. ${ }^{2}$

Clinical problems among homebound elderly are often multifaceted. It has been reported that $40.8 \%$ of homebound elderly people have 2-3 comorbid conditions, while $31.9 \%$ experience four or more comorbid conditions. ${ }^{3}$ The complexities arising from comorbid conditions often make it more difficult for elderly people to seek necessary medical help. Medication is another focus that needs to be considered. Some medication-related risk factors are associated with poor health outcomes, including lack of routine brown bag review, which means encouraging patients to bring all their medicines and supplements in a bag for the clinician to review; therapeutic duplication; multiple prescribers; multiple storage locations; expired medications; and poor adherence. ${ }^{4}$ How medicinal reconciliation works towards optimal prescription is an important topic.

Medicinal reconciliation may help decrease duplicate prescriptions, prescription errors, potential inappropriate medications and adverse medication reactions.

Without easily accessible primary care, older homebound adults often turn to less ideal episodic alternatives, such as emergency department visits and hospitalisations in times of health crises. ${ }^{2}$ This cycle repeats itself due to a lack of accessible primary care follow up. Hospitalisation frequently leads to loss of capacity for independent living and rapid functional deterioration, which renders these patients at high risk of institutionalisation.

Many countries have developed new care models to address access-to-care deficiencies and to postpone adverse health trajectories. ${ }^{2,5}$ Home-based care has been on a constant rise. ${ }^{6-8}$ For example, the Integrated Home Healthcare Program of the Taiwanese National Health Insurance (NHI) has been in practice since February 2016. The long-term purpose is to improve accessibility, and therefore, to decrease outpatient and emergency room visits and hospitalisation. Direct pharmacovigilance at home is also possible. This service is recognised widely by the public. ${ }^{9,10}$

By providing home healthcare, it was hoped that participants' healthcare utilisation, including outpatient visits, emergency care use, hospital admissions and prescription medications, might decrease. The outcome pattern could differ with disease stage. It was postulated that changes in healthcare utilisation might be different, given the level of home care services provided to patients with different disease stages. The aim of this study was to report changes in healthcare utilisation after enrolment into the home healthcare program. 


\section{Methods}

\section{Study design}

This was a multicentre follow-up study. Data on outpatient visits, emergency care use, hospitalisation and number of prescription medications of the participants of the Integrated Home Healthcare Program in Taiwan were obtained from electronic records of 12 medical institutions in Taipei City.

The observation period was from 1 January 2015 to 30 September 2016. Enrolment date into the program was defined as the index date. Mirror analysis was performed, and participants' healthcare utilisation and prescription medications before and after enrolment were compared. This study was approved by the Institutional Review Board of Taipei City Hospital (TCHIRB-10511101-E).

\section{Participants}

Participants of the Integrated Home Healthcare Program at Taipei City Hospital, Renai Branch, were enrolled between February and September 2016. A total of 415 patients were eligible for the program. Of these, 100 refused to receive home healthcare. Another 22 and 47 cases had duplicate or missing data, respectively. In total, there were 246 participants, as shown in Figure 1. For comparison purposes, the research group extended the observation period backwards to 2015, before enrolment (index date). Therefore, the look-back period (number of days) was not the same for all the participants.

Participants met the following inclusion criteria: 1) explicit medical needs; 2) homebound, which was defined as unable to leave one's house due to frailty or illness; however; with the help of a caregiver, and with the use of a wheelchair, paratransit service bus or ambulance, participants could still attend hospital appointments; and 3) the participant's home was within the catchment area $(10 \mathrm{~km})$ of the medical institution providing the service. Exclusion criteria included patient refusal to participate and residents of long-term care facilities. Oral and written informed consent was obtained prior to study commencement.
Participants were further divided into three subgroups according to the severity of their disease and different levels of service provided: home healthcare (step 1), advanced home healthcare (step 2), and palliative care at home (step 3). Step 1 participants did not have any inserted tubes $(\mathrm{n}=87)$. Step 2 participants had some form of inserted tubes, such as a nasogastric tube, tracheal tube or Foley catheter $(n=139)$. Step 3 participants were at the end stage of their disease and had a life expectancy of less than six months $(n=20)$. According to the Taiwanese NHI, these subgroups (steps 1-3) belong to different billing systems.

\section{Intervention}

The medical team comprised a physician and a nurse or a paramedical. A pharmacist, nutritionist, psychologist, social worker and physical or occupational therapist visited patients on an as-needed basis. The medical team visited patients at least every two months, and at most, every two weeks, depending on individual needs. Three steps of medical service were provided according to disease severity.
Step $1(n=87)$ : Home healthcare for people living at home with definite medical needs who cannot go to the hospital due to difficulties in transportation.

Step 2 ( $n=139)$ : Advanced home healthcare - for people meeting step 1 criteria who had certain inserted tubes, such as a nasogastric tube, tracheal tube or Foley catheter. Home healthcare services included changing of these tubes.

Step $3(n=20)$ : Palliative care at home for people at an irreversible stage of their disease and with a life expectancy of less than six months.

The medical care included:

- physician's visit - history taking, physical examination, clinical diagnosis and treatment, including prescription of medications. Simple procedures and health counselling were also provided

- nurse's visit - home nursing care. Simple procedures, such as blood tests, wound care or changing tubes, were provided

- visits by other multidisciplinary team members were provided on an as-needed basis. ${ }^{10}$

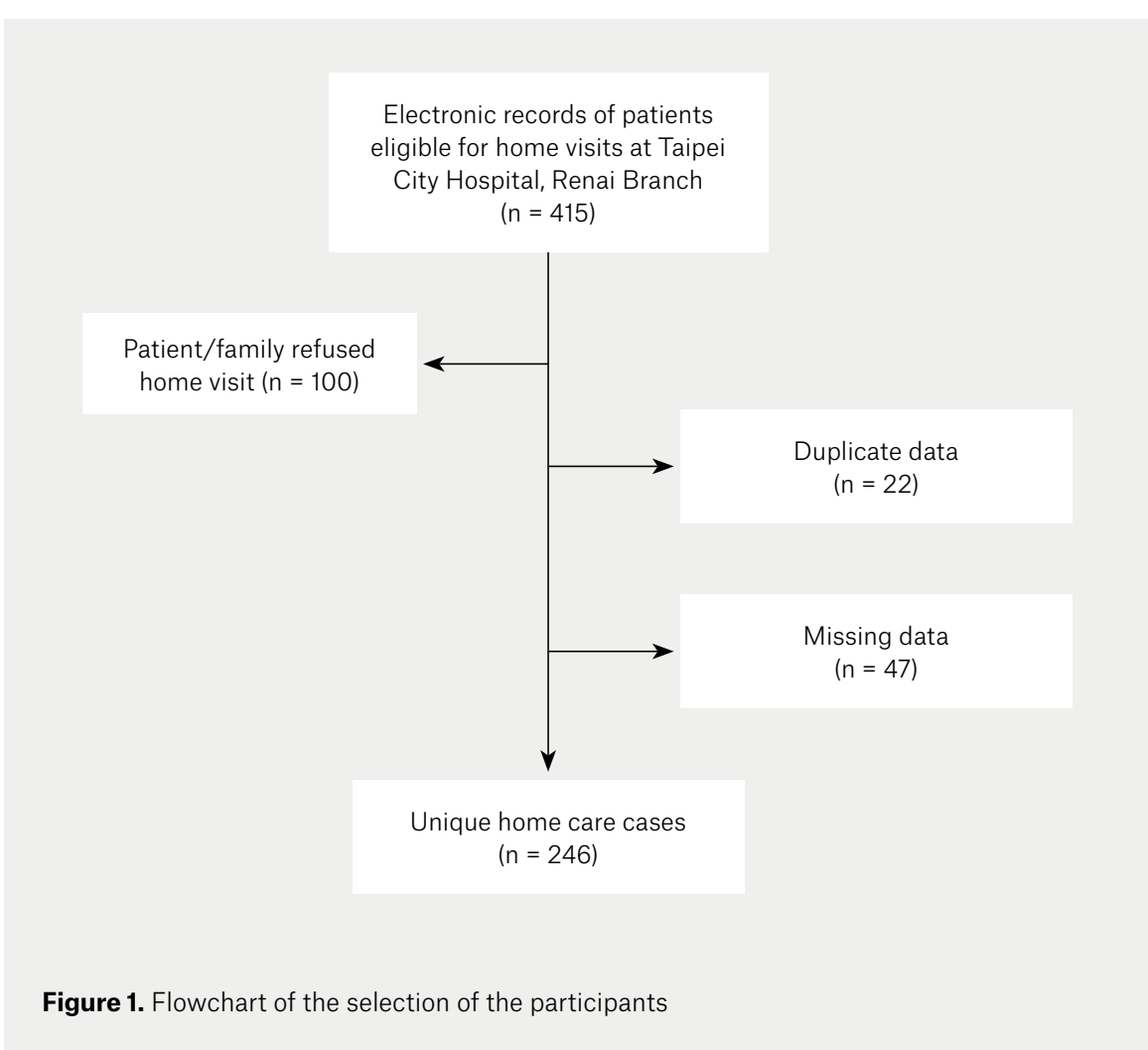


Physicians play a pivotal role in the complex medical care of this homebound and vulnerable population. ${ }^{11}$ Individualised care plans were developed for the patients. Throughout the care period, patients were continuously evaluated and referred to other health professionals if their health status changed.

During home visits, medications taken were reviewed (pharmacovigilance). This helps physicians monitor patients' adherence. ${ }^{12}$ This included a review of multiple medications prescribed from different medical institutions, as the Taiwanese NHI system allows people to visit any specialty clinic directly without the need to see a general practitioner (gatekeeper) first.

\section{Measurements}

Basic demographic data were collected at the first home visit. Health and functional status were assessed by clinicians directly during home visits. Electronic medical records of the 12 centres in Taipei City were linked, which provided information about healthcare utilisation (inpatient, outpatient and emergency visits) and prescription of medications. Additional health data, such as medication adherence and additional inpatient and outpatient and emergency visits, were collected during home visits. Diagnoses were made using the International Classification of Diseases-10 codes. Only five main diagnoses were recorded, because the NHI outpatient billing system only allows for a maximum of five diagnoses per visit. Functional status was assessed using the Barthel activities of daily living (ADL) index. Total, severe and partial/non-dependence were defined as Barthel scores of 0-20, 21-60 and 61-100, respectively. Disability referred to vision, hearing, language, limb or vital organ impairment; mental retardation; dementia; or vegetative state. Doctors visited per year referred to the number of doctors in outpatient clinics a patient visited in a year, as the Taiwanese NHI does not require seeing a gatekeeper doctor, primary care physician or family physician before seeking a specialist's help. Therefore, Taiwanese people are free to see any specialist (eg a neurosurgeon) at any time without the need for referral. Mortality rate after the index date was calculated, dividing mortality by personyears of observation.

To facilitate comparison before and after the index date, rates per year were presented instead of simply counts of outpatient visits, emergency room visits, hospital admissions and admission days. Therefore, outpatient/emergency room visits per year, hospital admissions per year and admission days per year were calculated.

Similarly, rates per year were presented for centres, specialties and doctors visited. Some physicians practised different specialties in different office hours and were counted as one doctor and multiple specialties. Medications were defined as the mean number of medications taken concomitantly during the observation period. Medications were classified using the anatomical therapeutic chemical codes. For comparison purposes, injection medications were not included in the analysis because the home care services mainly allow for the prescription of oral medications only. All this information came from a combination of electronic health records and direct observation. Healthcare utilisation and oral medications of the participants before and after program enrolment were analysed.

\section{Statistical analysis}

The research group conducted analyses stratified by sex and service step (ie different disease stages). SPSS version 21.0 was used. A $P$ value $<0.05$ for two-tailed tests was considered statistically significant. Paired Student's $t$-test and chi-squared tests were used as appropriate. Wilcoxon signed rank test was used when the data were not normally distributed (skewed). Fisher's exact test was used when the numbers in the cells were less than five.

\section{Results}

The mean age was 85.53 years (standard deviation [SD]: 10.0 ), and $52 \%$ of the participants were men. The majority received step 2 care (56.5\%; Table 1 ). Survival rates differed among participants of different care steps $(P<0.001)$, but not between men and women $(P=0.710)$. The one-year mortality rates for steps 1, 2 and 3 participants were $0.01,0.34$ and 1.83 , respectively; that is, the mean survival time for step 3 (palliative care) participants was approximately six months. The main diagnoses and medications of the participants are presented in Appendices 1 and 2, respectively (available online only).

Table 2 shows the healthcare utilisation of the participants before and after enrolment. The number of emergency room visits per year did not change significantly, although there was an increase in annual outpatient visits (from 13.7 to 15.3 visits/year). The number of admissions per year decreased for step 1 , but increased for step 3 participants (from 0.9 to 0.7 and from 1.7 to 2.7 admissions/year, respectively). There was a decrease in admission days per year after enrolment into the program (from 17.5 to 15 days/year).

Tables 3 and 4 show the pattern of outpatient services and medications used by the participants. Medical institutions visited per year increased (from 1.13 to 1.33 centres per year), but specialties and doctors visited per year decreased (from 3.81 to 2.57 specialties per year and from 3.76 to 2.31 doctors per year, respectively). Mean medications of the participants also increased (from 3.3 to 4.1 ).

\section{Discussion}

The impact of home healthcare service on healthcare resource utilisation and prescription medications in Taiwan was reported in the present study. Participants had fewer admission days, more outpatient visits and more oral medications after receiving home healthcare.

The researchers observed that after the implementation of the home healthcare program, overall admission times did not change significantly, but admission days decreased. Nabagiez et al reported that the home healthcare program reduced the 30 -day readmission rate by $41 \%$ in two years. ${ }^{13}$ Ju et al suggested that participants who did not use home visit nursing services had a higher risk of hospitalisation, particularly those with 
Table 1. Participants' baseline characteristics $(n=246)$

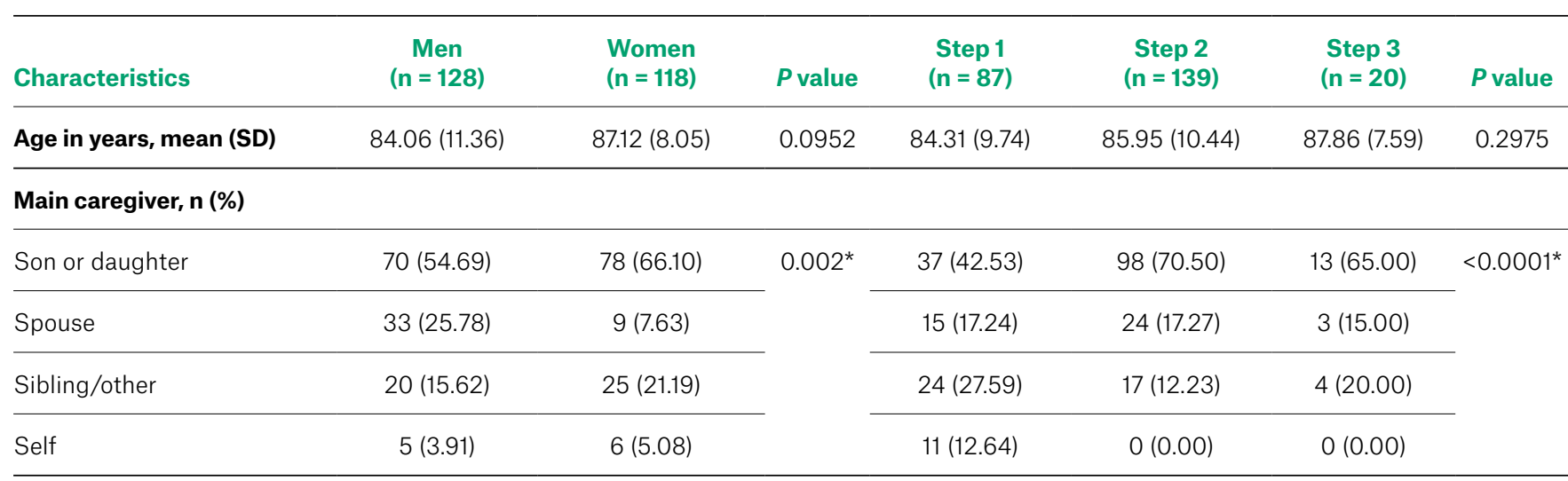

Consciousness, n (\%)

\begin{tabular}{lllllll}
\hline Clear & $71(55.47)$ & $50(42.37)$ & $0.0401^{*}$ & $72(82.76)$ & $42(30.22)$ & $7(35.00)$ \\
\cline { 1 - 2 } Unclear & $57(44.53)$ & $68(57.63)$ & & $15(17.24)$ & $97(69.78)$ & $13(65.00)$ \\
\hline
\end{tabular}

Expression, $\mathbf{n}(\%)$

\begin{tabular}{|c|c|c|c|c|c|c|c|}
\hline $\begin{array}{l}\text { Able to communicate } \\
\text { verbally }\end{array}$ & $83(64.8)$ & 67 (56.78) & 0.079 & 76 (87.36) & $63(45.32)$ & $11(55.00)$ & $<0.0001^{*}$ \\
\hline Gestures only & 14 (10.9) & $8(6.78)$ & & $8(9.20)$ & 14 (10.07) & $0(0.00)$ & \\
\hline $\begin{array}{l}\text { Unable to express/not } \\
\text { evaluable }\end{array}$ & $31(24.2)$ & $43(36.44)$ & & $3(3.45)$ & $62(44.60)$ & $9(45.00)$ & \\
\hline
\end{tabular}

Functional status, $\mathbf{n}(\%)$

\begin{tabular}{|c|c|c|c|c|c|c|c|}
\hline Total dependence & $92(71.88)$ & 84 (71.19) & 0.967 & $31(35.63)$ & 129 (92.81) & $16(80.00)$ & $<0.0001^{*}$ \\
\hline Heavy dependence & $32(25.00)$ & $31(26.27)$ & & $49(56.32)$ & $10(7.19)$ & $4(20.00)$ & \\
\hline
\end{tabular}

Respiratory aids, $\mathbf{n}(\%)$

\begin{tabular}{|c|c|c|c|c|c|c|c|}
\hline Oxygen/tracheostomy & $17(13.28)$ & $14(11.86)$ & & $3(3.45)$ & $20(14.39)$ & $5(25.00)$ & \\
\hline \multicolumn{8}{|l|}{ Feeding method, n (\%) } \\
\hline Oral & $62(48.44)$ & $56(47.46)$ & 0.8779 & $87(100.00)$ & $22(15.83)$ & $9(45.00)$ & $<0.0001^{*}$ \\
\hline \multicolumn{8}{|c|}{ Urination and defecation, n (\%) } \\
\hline Continent & $52(40.63)$ & $45(38.14)$ & 0.6898 & 77 (88.51) & $16(11.51)$ & $4(20.00)$ & $<0.0001^{*}$ \\
\hline $\begin{array}{l}\text { Foley catheter or } \\
\text { incontinence }\end{array}$ & 76 (59.38) & $73(61.86)$ & & $10(11.49)$ & $123(88.49)$ & $16(80.00)$ & \\
\hline
\end{tabular}


greater comorbidities. ${ }^{14}$ Experiences in Australia demonstrated that home visits can decrease use of ambulance and emergency department services. ${ }^{15}$ A possible explanation was that home visits could prevent ADL and care needs deterioration up to one year after program completion. ${ }^{16}$ Medical personnel can enhance the skills of caring of family caregivers. Home visits serve as a support to caregivers, provide diagnostic information and help the family with the decision as to when hospitalisation is appropriate. ${ }^{17}$ Home services improve the quality of self-care and address hidden disease burden and unmet care needs. ${ }^{18,19}$ A reduction in bed days has also been demonstrated at Monash Watch Hospital, Australia, after telehealth and coaching implementation; the improvement was more prominent in the intervention group in Martin et al's study. ${ }^{20}$

The research group found that providing home services resulted in a decrease in admission days but an increase in outpatient visits, which was an unexpected finding. The effect on emergency room visits was not significant. The program reduced hospital admissions and diverted healthcare utilisation to outpatient visits. However, hospital admission overall was not significantly reduced and could be confounded by disease severity. After stratification, step 1 participants had fewer hospitalisations after home healthcare provision. Step 2 participants did not have a significant change in admission times, but had fewer admission days. Step 3 participants had more hospitalisations after the index date.

It is unclear why outpatient visits increased. The Taiwanese NHI system allows beneficiaries to access any hospital and any specialty as they wish. One possible reason could be that, after joining the program, participants' loyalty towards the hospital providing home services increased, and therefore, shifted and concentrated their regular clinic visits to the institutions involved in the program. Another possibility was that the home

\section{Table 2. Healthcare utilisation of the participants before and after enrolment into the program}

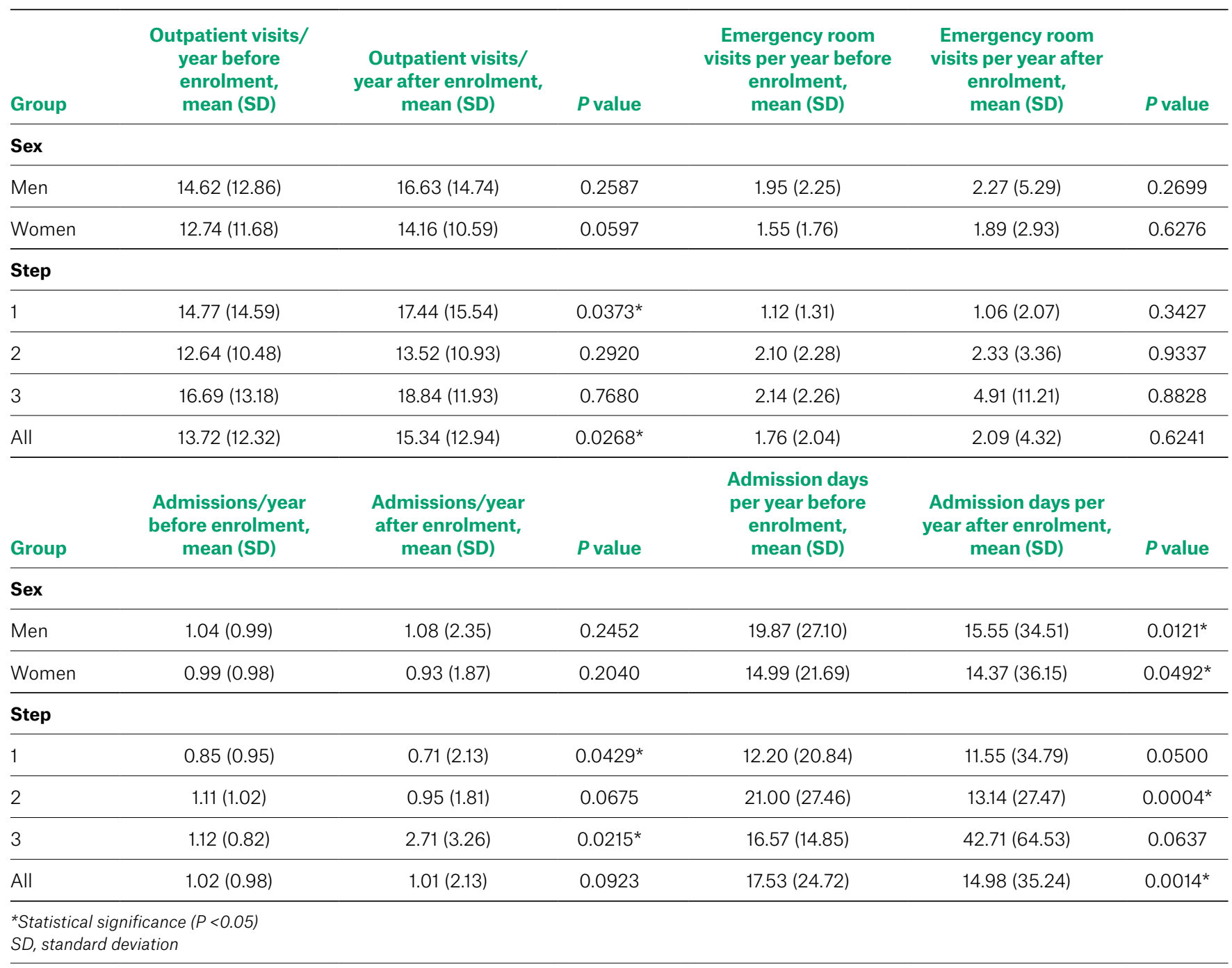


visiting staff directed patients to their own institution instead of other private practitioners.

The analysis of outpatient visits further supported this hypothesis. Although outpatient visits and medical institutions (centres) visited increased, specialties and doctors visited after enrolment into the program decreased uniformly across subgroups. Joining the program might increase patients' willingness to access service institutions, while reducing their behaviour of seeing multiple doctors.

Polypharmacy is common among the elderly and is an important risk factor for adverse medication reactions and poor health outcomes. ${ }^{4,21}$ Home visits enable a better understanding of patients' medication adherence, and therefore, are associated with comprehensive medical care. ${ }^{12}$ The mean number of medications increased after enrolment into the program. Physicians' pharmacovigilance might have unified participants' previous regular prescriptions. However, necessary new medications might have been prescribed after thorough evaluation of the patient at home in response to previous unmet needs. Nevertheless, the underlying cause of this increase in prescription medications warrants future study.

\section{Strengths}

This study has several strengths. Few studies have discussed changes in

Table 3. Outpatient services used by the participants (rates/year are shown)

\begin{tabular}{|c|c|c|c|c|c|c|c|c|c|}
\hline Group & $\begin{array}{c}\text { Centres } \\
\text { visited/year } \\
\text { before index } \\
\text { date, } \\
\text { mean (SD) }\end{array}$ & $\begin{array}{c}\text { Centres } \\
\text { visited/year } \\
\text { after index } \\
\text { date, } \\
\text { mean (SD) }\end{array}$ & $P$ value & $\begin{array}{c}\text { Specialties } \\
\text { visited/year } \\
\text { before index } \\
\text { date, } \\
\text { mean (SD) }\end{array}$ & $\begin{array}{c}\text { Specialties } \\
\text { visited/year } \\
\text { after index } \\
\text { date, } \\
\text { mean (SD) }\end{array}$ & $P$ value & $\begin{array}{c}\text { Doctors } \\
\text { visited/year } \\
\text { before index } \\
\text { date, } \\
\text { mean (SD) }\end{array}$ & $\begin{array}{c}\text { Doctors } \\
\text { visited/year } \\
\text { after index } \\
\text { date, } \\
\text { mean (SD) }\end{array}$ & $P$ value \\
\hline \multicolumn{10}{|l|}{ Sex } \\
\hline Men & $1.09(0.319)$ & $1.34(0.525)$ & $<0.001^{*}$ & $4.08(2.77)$ & 2.64 (1.499) & $<0.001^{*}$ & 4.03 (3.718) & $2.35(1.616)$ & $<0.001^{*}$ \\
\hline \multicolumn{10}{|l|}{ Step } \\
\hline 1 & $1.15(0.485)$ & $1.67(0.543)$ & $<0.001^{*}$ & 4.63 (3.118) & $3.11(1.826)$ & $0.001^{*}$ & 4.47 (3.898) & $2.42(1.848)$ & $<0.0001^{*}$ \\
\hline 2 & $1.10(0.324)$ & $1.15(0.38)$ & 0.21 & $3.33(2.336)$ & $2.23(1.358)$ & $<0.0001$ & 3.45 (3.185) & $2.28(1.684)$ & $<0.0001^{*}$ \\
\hline
\end{tabular}

*Statistical significance $(P<0.05)$

$S D$, standard deviation

Table 4. Oral medications used by the participants

\begin{tabular}{|c|c|c|c|c|c|}
\hline Group & $\begin{array}{l}\text { Medications before } \\
\text { enrolment (range) }\end{array}$ & $\begin{array}{l}\text { Medications after } \\
\text { enrolment (range) }\end{array}$ & $\begin{array}{l}\text { Medications before } \\
\text { enrolment, mean (SD) }\end{array}$ & $\begin{array}{l}\text { Medications after } \\
\text { enrolment, mean (SD) }\end{array}$ & $P$ value \\
\hline \multicolumn{6}{|l|}{ Sex } \\
\hline Women & $0-12.35$ & $0-14.30$ & 3.05 (3.02) & $3.84(3.10)$ & $0.0003^{*}$ \\
\hline 1 & $0-12.60$ & $0-16.20$ & 2.89 (3.02) & $4.08(3.03)$ & $<0.0001^{*}$ \\
\hline 2 & $0-13.10$ & $0-12.66$ & $3.30(2.96)$ & $3.94(3.10)$ & $0.0071^{*}$ \\
\hline 3 & 0-11.89 & $0-14.30$ & 4.46 (3.98) & $5.72(4.10)$ & 0.1415 \\
\hline All & $0-13.10$ & $0-16.20$ & 3.25 (3.08) & 4.13 (3.18) & $<0.0001^{*}$ \\
\hline
\end{tabular}

*Statistical significance $(P<0.05)$

$S D$, standard deviation 
healthcare utilisation (eg emergency care, outpatient and inpatient visits, as well as medications) after the provision of home healthcare in such a comprehensive way. To the researchers' knowledge, this is the first study to do so. Temporal sequence is the strongest indicator of causality. Although not a randomised controlled trial, the before/after design is the best feasible design to evaluate the short-term effect of the intervention. The research group had perfect matching of the study participants before and after the intervention. The study reported participants' doctorseeking behaviours, which has been rarely discussed in previous studies. The researchers analysed changes across different disease stages (steps 1-3). To the best of the researchers' knowledge, the present study is this first to do so.

\section{Limitations}

This was a relatively new service model, and the case numbers were limited. This was a pre/post evaluation and was, therefore, prone to regression to the mean. The impact of a counterbalancing deterioration factor in vulnerable health trajectories also needs to be accounted for. There was no control group. However, this study compared the healthcare utilisation of the same person so that confounding was minimised. It was not able to consider the effect of ageing, even though there was only a one-year increment. Although the study presented healthcare utilisation in rates per year, possible differences across seasons were not accounted for. ADL was measured on the index date and no longitudinal ADL was available. Multiple comparisons were made in this study, which might increase type 1 errors. Nevertheless, this study did have some unexpected findings. For example, emergency room visits were not decreased. This could be regarded as a pioneer study in Taiwan and viewed as a sentinel report that may be applicable to other countries.

\section{Conclusion}

Care of elderly homebound patients is a challenge for the healthcare system. ${ }^{17}$ Home visits may be beneficial for homebound older people in terms of decreasing days of hospital admission but does not decrease medication use or outpatient or emergency room visits. In this study, improved continuity of care was associated with fewer doctor visits. Further large-scale, randomised controlled trials with control groups are required to confirm the findings of the present study. How patients and caregivers rate changes to quality of life will be the next research focus. Differences in outcomes, according to frequency of home healthcare visits and services, in the future will also be a focus of future research.

\section{Implications for general practice}

- Home healthcare services are increasing in many countries in response to global ageing and the goal to age in place.

- In a multicentre study, it was observed that, after the provision of home healthcare services, there was a decrease in hospital admission days.

- Home healthcare services did not decrease outpatient or emergency room visits.

- Oral medications increased after the provision of home healthcare services.

\section{Authors}

Tai-Yin Wu MD, PhD, Attending Physician, Department of Family Medicine, Taipei City Hospital, Zhongxing Branch, Taiwan; Assistant Professor, Institute of Epidemiology and Preventive Medicine,

National Taiwan University, Taiwan; Adjunct Assistant

Professor, University of Taipei, Taiwan; Assistant

Professor, National Taipei University of Nursing and

Health Sciences, Taiwan

Sheng-Huang Hsiao MD, PhD, Attending Physician,

Department of Neurosurgery, Taipei City Hospital,

Renai Branch, Taiwan

Sheng-Jean Huang MD, Attending physician,

Department of Neurosurgery, Taipei City Hospital,

Taiwan

Kuan-Liang Kuo MD, PhD, Attending Physician, Department of Family Medicine, Taipei City Hospital, Renai Branch, Taiwan

Lin-Chung Woung MD, PhD, Attending physician, Department of Ophthalmology, Taipei City Hospital,

Renai Branch, Taiwan

Fang-Chun Chen MD, Attending Physician,

Department of Family Medicine, Taipei City Hospital,

Renai Branch, Taiwan

Competing interests: None.

Funding: None.

Provenance and peer review: Not commissioned, externally peer reviewed.

Correspondence to:

DAW15@tpech.gov.tw

\section{Acknowledgements}

The authors thank Mr Hua Chun for his help with the data analysis; Ms Hsiao-Ting Lin, Ms Yi-Ling Chen and $\mathrm{Mr}$ Tzu-Heng Fu for their managerial support; and the Information Technology Office of Taipei City Hospital for providing the electronic medical records.

\section{References}

1. Ministry of Health and Welfare, Taiwan (ROC). Report of the senior citizen condition survey 2013. Taiwan: Ministry of Health and Welfare, 2014. Available at www.mohw.gov.tw/dl-4707-e91899c0d62d-49d9-9dbe-4a2def8e516c.html [Accessed 9 March 2021]

2. Stall N, Nowaczynski M, Sinha SK. Back to the future: Home-based primary care for older homebound Canadians: Part 1: Where we are now. Can Fam Physician 2013;59(3):237-40.

3. Qiu WQ, Dean M, Liu T, et al. Physical and mental health of the homebound elderly: An overlooked population. J Am Geriatr Soc 2010; 58(12):2423-28. doi: 10.1111/j.1532-5415.2010.03161.x.

4. Sorensen L, Stokes JA, Purdie DM, Woodward M, Roberts MS. Medication management at home: Medication-related risk factors associated with poor health outcomes. Age Ageing 2005;34(6):626-32. doi: 10.1093/ageing/afi202.

5. Lin YD, Huang PY, Huang CJ, et al. The role and future of integration Chinese and western medicine in hospital-based accountable family physicians system. Taipei City Med J 2016;13(1):106-11. doi: 10.6200/tcmj.2016.13.1.11.

6. Stijnen MM, Van Hoof MS, Wijnands-Hoekstra IY, et al. Detected health and well-being problems following comprehensive geriatric assessment during a home visit among community-dwelling older people: Who benefits most? Fam Pract 2014;31(3):333-40. doi: 10.1093/fampra/cmu015.

7. Ruiz S, Snyder LP, Rotondo C, Cross-Barnet C, Colligan EM, Giuriceo K. Innovative home visit models associated with reductions in costs, hospitalizations, and emergency department use. Health Aff (Millwood) 2017;36(3):425-32. doi: 10.1377/hlthaff.2016.1305

8. Yang M, Thomas J, Zimmer R, Cleveland M, Hayashi JL, Colburn JL. Ten things every geriatrician should know about house calls. J Am Geriatr Soc 2019;67(1):139-44. doi: 10.1111/ jgs.15670.

9. Chung $\mathrm{CH}$, Huang LY, Sun WJ. Hospital-based accountable family physicians system: The pilot study of community-based integrated care program. Taipei City Med J 2016;13(1):72-79. doi: 10.6200/tcmj.2016.13.1.07.

10. Chen YC, Chen MJ, Yang RJ. Satisfaction on hospital-based accountable family physicians system in elderly living alone. Taipei City Med J 2016;13(1):95-105. doi: 10.6200/tcmj.2016.13.1.10.

11. Zweig SC, Popejoy LL, Parker-Oliver D, Meadows SE. The physician's role in patients nursing home care: 'She's a very courageous and lovely woman. I enjoy caring for her'. JAMA 2011;306(13):1468-78. doi: 10.1001/ jama.2011.1420.

12. Liang YH, Chen M-J, Wu S-C. Pharmacists' assessment of medication profile in elders with high risk of dementia in hospital-based family physicians system. Taipei City Med J 2016;13(2):151-61. doi: 10.6200/tcmj.2016.13.2.05.

13. Nabagiez JP, Shariff MA, Molloy WJ, Demissie S, McGinn JT. Cost analysis of physician assistant home visit program to reduce readmissions after cardiac surgery. Ann Thorac Surg 2016;102(3):696-702. doi: 10.1016/j. athoracsur.2016.03.077. 
14. Ju YJ, Lee HJ, Kim W, Lee SA, Han K-T, Park E-C. Association between home-visit nursing utilization and all-cause hospitalization among long-term care insurance beneficiaries: A retrospective cohort study. Int J Nurs Stud 2017;75:93-100. doi: 10.1016/j.ijnurstu.2017.07.016.

15. McRae I, Pham M. When is a GP home-visit program financially viable? Aust J Prim Health 2016;22(6):554-58. doi: 10.1071/PY15074.

16. Kono A, Izumi K, Yoshiyuki N, Kanaya Y, Rubenstein LZ. Effects of an updated preventive home visit program based on a systematic structured assessment of care needs for ambulatory frail older adults in Japan: A randomized controlled trial. J Gerontol A Biol Sci Med Sci 2016;71(12):1631-37. doi: 10.1093/gerona/ glw068.

17. Vinker S, Nakar S, Weingarten MA. Home visits to the housebound patient in family practice: A multicenter study. Israeli General Practice Research Network. Isr Med Assoc J 2000;2(3):203-06.

18. Yeh $\mathrm{CC}$, Hung RP, Chung $\mathrm{H}$, et al. The effect of home visit on blood sugar and lipid profile in diabetic patients. Taipei City Med J 2016;13(1):41-46. doi: 10.6200/tcmj.2016.13.1.04.

19. Han D, Wilks A, Mattke S. Detection of undiagnosed disease in Medicare beneficiaries after a clinical home visit. Popul Health Manag 2017;20(1):41-47. doi: 10.1089/pop.2015.0187.

20. Martin C, Hinkley N, Stockman K, Campbell D. Capitated telehealth coaching hospital readmission service in Australia: Pragmatic controlled evaluation. J Med Internet Res 2020;22(12):e18046. doi: 10.2196/18046.

21. Wu TY, Jen MH, Bottle A, et al. Ten-year trends in hospital admissions for adverse drug reactions in England 1999-2009. J R Soc Med 2010;103(6):239-50. doi: 10.1258/ jrsm.2010.100113. 